Ophthalmologe $2018 \cdot 115: 114-122$

DOI 10.1007/s00347-017-0497-8

Online publiziert: 24. April 2017

(c) Der/die Autor(en) 2017. Dieser Artikel ist eine Open-Access-Publikation.

CrossMark
P. Bhogal' $\cdot$ M. Aguilar Pérez' • G. Sauder ${ }^{2} \cdot$ H. Bäzner ${ }^{3} \cdot$ O. Ganslandt ${ }^{4} \cdot$ H. Henkes ${ }^{1,5}$

${ }^{1}$ Neuroradiologische Klinik, Neurozentrum, Klinikum Stuttgart, Stuttgart, Deutschland

${ }^{2}$ Charlottenklinik, Stuttgart, Deutschland

${ }^{3}$ Neurologische Klinik, Neurozentrum, Klinikum Stuttgart, Stuttgart, Deutschland

${ }^{4}$ Neurochirurgische Klinik, Neurozentrum, Klinikum Stuttgart, Stuttgart, Deutschland

${ }^{5}$ Medizinische Fakultät, Universität Duisburg-Essen, Essen, Deutschland
Aneurysmen der Hirngefäße sind eine vergleichsweise häufige Erkrankung mit einer Prävalenz von 2-3\% bei Patienten ohne spezifische Risikofaktoren. Die Mehrzahl dieser Aneurysmen ist klein und an den Gefäßen der vorderen Zirkulation lokalisiert [1]. Die Prävalenz intrakranieller Aneurysmen ist bei Frauen höher und nimmt mit zunehmendem Alter zu. Verschiedene Risikofaktoren gehen mit einem gehäuften Auftreten intrakranieller Aneurysmen einher. Dazu gehören Nikotinabusus, exzessiver Alkoholkonsum, arterielle Hypertonie und ein oder mehrere Blutsverwandte mit erlittener Subarachnoidalblutung, aber auch eine selbst bereits erlittene Subarachnoidalblutung und Erkrankungen wie die autosomal-dominante polyzystische Nierenerkrankung.

Paraophthalmische Aneurysmen waren im International Subarachnoid Aneurysm Trial(ISAT) mit über $10 \%$ vertreten [2], wurden aber in anderen Studien mit einer Häufigkeit von bis zu $25 \%$ aller intrakraniellen Aneurysmen angegeben [3]. Paraophthalmische Aneurysmen repräsentieren ungefähr $0,5 \%$ aller rupturierten Aneurysmen [4, 5]. Wie andere intradurale Aneurysmen stellen sie einen Risikofaktor für das Auftreten einer Subarachnoidalblutung dar, auch wenn einzelne Studien die Vermutung nahelegen, dass das Blutungsrisiko bei paraophthal-

Die Ergebnisse wurden in einem Vortrag auf dem DOG-Kongress 2016 in Berlin präsentiert. mischen Aneurysmen etwas geringer ist als bei Aneurysmen in anderen intraduralen Lokalisationen $[6,7]$.

Unabhängig von der Aneurysmaruptur kann es zu einer Kompression des Sehnervs mit Visusminderung kommen, was ab einer Größe des Aneurysmafundusvon 5 mm oder mehr beobachtet wird [8]. Die Behandlungsempfehlung basiert auf der Größe und Morphologie des Aneurysmas und auf weiteren Faktoren, wie z. B. einem nachgewiesenen Aneurysmawachstum oder bereits eingetretenen Sehstörungen. Eine Behandlung ist darüber hinaus nach einer Aneurysmaruptur mit akuter subarachnoidaler oder intrazerebraler Blutung erforderlich. Die Behandlung kann entweder mikrochirurgisch oder endovaskulär erfolgen. In dieser Übersicht stellen wir die verschiedenen endovaskulären Behandlungsoptionen für solche Aneurysmen dar.

\section{Anatomische Aspekte}

Das Auge entwickelt sich embryologisch aus dem Dienzephalon, und entsprechend erfolgt seine Blutversorgung ähnlich wie die des Gehirns. Die A. ophthalmica wird häufig als eine Endarterie betrachtet, hat in Wirklichkeit aber eine Kollateralversorgung von der A. carotis externa. Wenn die A. ophthalmica langsam verschlossen wird, kann die A. carotis externa die Blutversorgung des Bulbus schrittweise übernehmen und durch den Erhalt der Versorgung der Retina und der choroidalen Gefäße trotz Ophthalmica-Verschluss eine Visusminderung vermeiden.

》) Die A. ophthalmica hat eine Kollateralversorgung von der

\section{A. carotis externa}

Das paraophthalmische Segment der A. carotis interna wird definiert als dasjenige Gefäßsegment, dass sich vom Ursprung der A. ophthalmica bis zum Ursprung des R. communicans posterior erstreckt ([9]; - Abb. 1).

Aneurysmen können überall an diesem Gefäßsegment entstehen. Eine hilfreiche anatomische Klassifikation wurde von Rouchaud et al. [10] vorgeschlagen (- Abb. 2). Dieses Klassifikationsschema stellt eine Beziehung zwischen der A. ophthalmica und dem Aneurysma selbst her:

- Typ A: Die A. ophthalmica entspringt aus dem Aneurysmasack.

- Typ B: Die A. ophthalmica entspringt am Aneurysmahals.

- Typ C: Das Aneurysma entspringt von der konkaven Seite des paraophthalmischen Segments der A. carotis interna. Diese Aneurysmen werden auch Aneurysmen der oberen Hypophysenarterie genannt.

- Typ D: Die A. ophthalmica ist nicht in das Aneurysma einbezogen, das Aneurysma entspringt distal des Abgangs der A. ophthalmica auf 


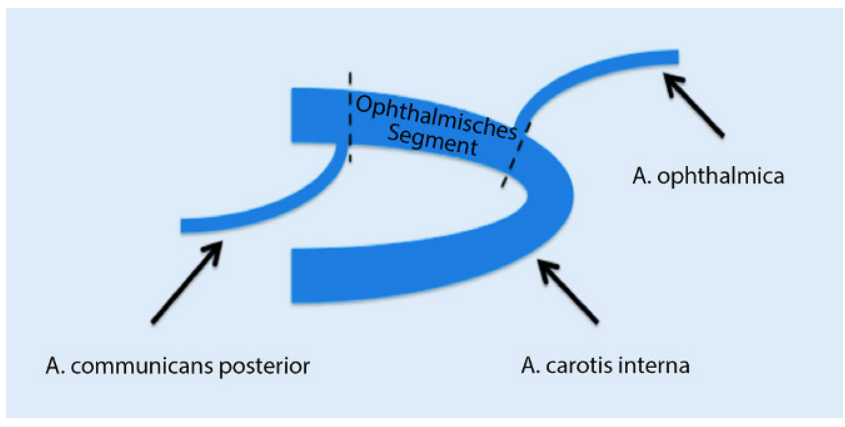

Abb. $1<$ Anatomische Klassifikation paraophthalmischer Aneurysmen der A. carotis interna
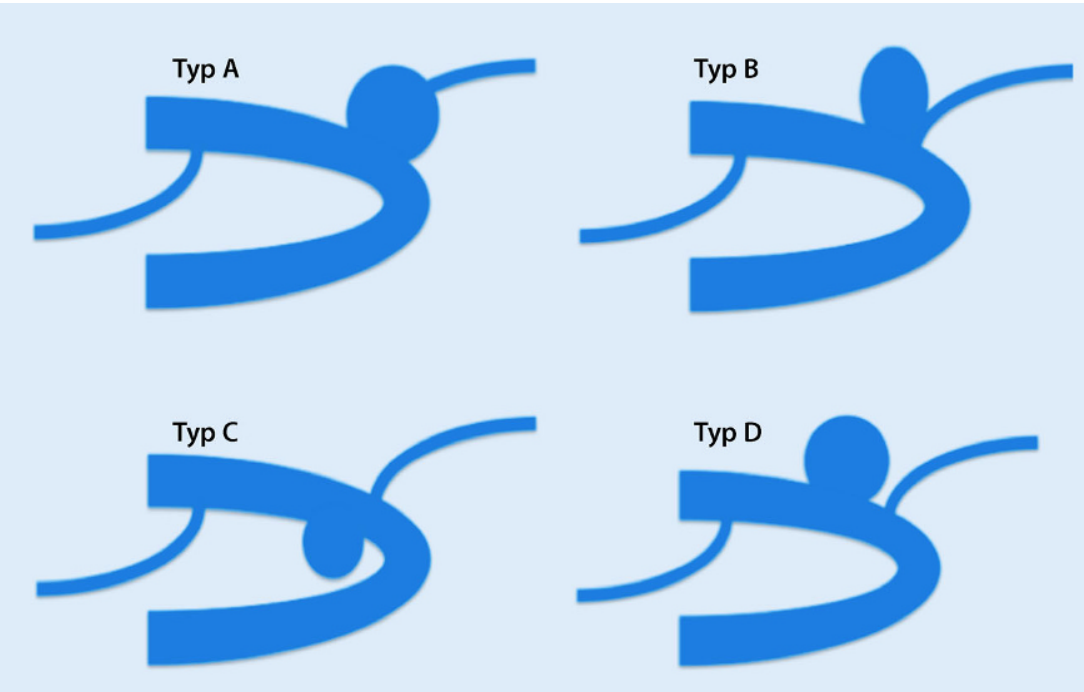

Abb. $2 \Delta$ Die anatomische Klassifikation von Aneurysmen in Bezug zur A. ophthalmica geht auf Rouchaud et al. zurück. Bei dem Typ-A-Aneurysma entspringt die A. ophthalmica aus dem Aneurysmafundus. Beim Typ-B-Aneurysma entspringt die A. ophthalmica in der Nähe des Aneurysmahalses. Typ-C-Aneurysmen entspringen auf der konkaven Seite der A. carotis interna, distant zum Ursprung $\operatorname{der}$ A. ophthalmica. Sie werden auch Aneurysmen der oberen Hypophysenarterie genannt. Beim Typ D entspringen die Aneurysmen auf dem vorderen und oberen Aspekt der A. carotis interna und sind ebenfalls entfernt vom Ursprung der A. ophthalmica lokalisiert (supraklinoidale Aneurysmen)

der konvexen Seite der A. carotis interna. Diese Aneurysmen werden auch supraklinoidale Aneurysmen genannt.

\section{Chirurgische Behandlung}

Paraophthalmische Aneurysmen sind chirurgisch erreichbar, und der Zugang erfolgt typischerweise über eine pterionale Kraniotomie. Um ausreichenden Zugang zum Aneurysma zu schaffen, ist die Entfernung des vorderen Klinoidfortsatzes häufig erforderlich. Dies kann auch notwendig sein, um Zugang zur proximalen A. carotis interna herzustellen. In der Phase der Clip-Aufbringung ist eine solche proximale Kontrolle für den Fall hilfreich, dass es zu einer in-

\section{Endovaskuläre Behandlung}

Seit der Publikation der wegweisenden ISAT-Studie [2] hat die endovaskuläre Behandlung intrakranieller Aneurysmen exponentiell zugenommen, und viele Institutionen haben mittlerweile eine Behandlungsstrategie übernommen, die endovaskuläre Verfahren vorrangig in Betracht zieht, um intrakranielle Aneurysmen zu behandeln. Die endovaskuläre Behandlung von Aneurysmen begann aber natürlich bereits vor der ISATStudie. Roy et al. [19] veröffentlichten ihre Ergebnisse mit der Behandlung von Aneurysmen im paraophthalmischen Segment der A. carotis interna mit Verwendung von ablösbaren Guglielmi Detachable Coils(GDC)-Spiralen im Jahr 1997. In dieser prospektiven Studie (26 Patienten, 28 Aneurysmen, von denen 4 zuvor operiert worden waren, aber chirurgisch nicht versorgt werden konnten) wurde ein vollständiger Verschluss bei $50 \%$ erreicht, und ein geringer Halsrest verblieb bei $39 \%$ dieser Aneurysmen. Die Autoren wiesen darauf hin, dass die Größe des Aneurysmahalses den besten Voraussagewert für das endgültige angiographische Ergebnis hatte. Angesichts dieser Herausforderungen breitbasiger Aneurysmen für die endovaskuläre Behandlung wurden spezifische Lösungen für diese Probleme gesucht. Dies bestand zuerst im sog. Ballon-Remodeling und einige Jahre später dann in der Verwendung selbstexpandierender Stents. Beide RemodelingTechniken wurden für die Behandlung paraophthalmischer Aneurysmen eingesetzt. Yavuz et al. [20] berichten über ihre Ergebnisse bei der Behandlung 8 paraophthalmischer Aneurysmen mit Stent-assistierter Coil-Okklusion. In dieser Patientenserie traten weder Mortalität noch Morbidität auf, und vollständiger oder nahezu vollständiger Aneurysmaverschluss wurde in 87,5\% erzielt. Weitere Gruppen haben ähnliche, herausragend gute Ergebnisse mit 87,5-100\% Verschlussrate bei nachfolgenden Angiographien erzielt [21-23]. Bei großen und riesenhaften paraophthalmischen Aneurysmen dagegen sind die mittelfristigen Verschlussraten nach 
Ophthalmologe 2018 · 115:114-122 DOI 10.1007/s00347-017-0497-8

(c) Der/die Autor(en) 2017. Dieser Artikel ist eine Open-Access-Publikation.

\section{P. Bhogal · M. Aguilar Pérez · G. Sauder $\cdot$ H. Bäzner $\cdot 0$. Ganslandt $\cdot$ H. Henkes}

\section{Management paraophthalmischer Aneurysmen. Übersicht der endovaskulären Behandlungsstrategien}

\section{Zusammenfassung}

Hintergrund. Aneurysmen der Hirngefäße sind relativ häufig und Aneurysmen des ophthalmischen Segments der A. carotis interna (ICA) machen ungefähr $10 \%$ der intrakraniellen Aneurysmen aus. Die Ruptur eines paraophthalmischen Aneurysmas kann zu einer unter Umständen lebensbedrohlichen intrakraniellen Blutung führen. Durch Druck auf den benachbarten $\mathrm{N}$. opticus kann eine Sehstörung verursacht werden.

Anatomische Aspekte. Das paraophthalmische Segment der A. carotis interna wird definiert als das Gefäßsegment, das sich vom Abgang der A. ophthalmica bis zum Ursprung des Ramus communicans posterior erstreckt. Aneurysmen können an der A. ophthalmica oder unabhängig davon an der ICA lokalisiert sein. Die unterschiedlichen anatomischen Strukturen können die Risiken der Behandlung beeinflussen.
Chirurgische Behandlung. Die mikrochirurgische Behandlung paraophthalmischer Aneurysmen stellt eine besondere Herausforderung für den Operateur wegen der tiefen Lokalisation und der komplexen umgebenden Strukturen sowie des N. opticus dar. Die Notwendigkeit der Entfernung des Klinoidfortsatzes kann zur technischen Herausforderung bei einer Operation werden. Endovaskuläre Behandlung. Zu den endovaskulären Behandlungsoptionen der paraophthalmischen Aneurysmen gehörten bisher die alleinige Coil-Okklusion, die CoilOkklusion mit Ballon-Remodeling und die Stent-assistierte Coil-Okklusion. Durch den Einsatz hämodynamisch aktiver Stents (sog. Flow Diverter) werden jetzt noch bessere Ergebnisse erzielt.

Schlussfolgerung. Die Behandlung paraophthalmischer Aneurysmen ist mit spezifischen
Risiken behaftet. Eine sorgfältige Abwägung vor jeder Intervention ist erforderlich. Rupturierte paraophthalmische Aneurysmen werden bevorzugt durch Coil-Okklusion endovaskulär behandelt. Durch die Flow Diverter-Implantation werden bei nicht rupturierten paraophthalmischen Aneurysmen jetzt noch bessere Ergebnisse erzielt. Die Komplikationsraten dieser Prozeduren sind insgesamt niedrig. Mögliche Komplikationen wie zerebrale Ischämie, intrakranielle Blutung aber auch Visusminderung bis zur ipsilateralen Erblindung müssen jedoch im Voraus bedacht und die Patienten müssen auf diese Gefahren hingewiesen werden.

Schlüsselwörter

Flow Diverter · A. ophthalmica · Stent - CoilOkklusion · Komplikationen

\section{Management of paraophthalmic aneurysms. Review of endovascular treatment strategies}

\section{Abstract}

Background. Aneurysms of the cerebral vessels are relatively common and aneurysms of the ophthalmic segment of the internal carotid artery (ICA) represent approximately $10 \%$ of intradural aneurysms.

Anatomical aspects. The ophthalmic segment of the ICA extends from the origin of the ophthalmic artery to the origin of the posterior communicating artery. Aneurysms can originate from the ophthalmic artery itself or directly from the ICA at a distance from the ophthalmic artery. The various anatomic configurations can alter the risks of treatment. Surgical treatment. Surgical treatment of aneurysms of the ophthalmic segment poses unique challenges for the neurosurgeon, given the deep location and complex nearby structures and optic nerve. The necessity to remove the clinoid process can make the operation technically challenging. Endovascular treatment. Endovascular treatment of paraophthalmic aneurysms includes coil occlusion, and also stent- or balloon-assisted coil occlusion. More recently, hemodynamically active flow diverter stents improved the treatment results.

Conclusion. The management of paraophthalmic aneurysms is associated with specific risks. Careful consideration of the available techniques is necessary before each invention.
Coil-occlusion remains the first choice for ruptured paraophthalmic aneurysms. For unruptured paraophthalmic aneurysms flow diversion offers improved results. The overall complication rates of these procedures are low; however, cerebral ischemia, intracranial hemorrhage and visual complications must be considered in advance and the patient must be informed of the risks.

\section{Keywords}

Flow diverter · Ophthalmic artery · Stents · Coil occlusion · Complications
Coil-Okklusion nicht zufriedenstellend (• Abb. 4).

Wie bereits oben erwähnt, gibt es eine Kollateralversorgung der A. ophthalmica, die von der A. carotis externa ausgeht. Dennoch sollte der akute Verschluss der A. ophthalmica vermieden werden, um keine Sehstörungen zu riskieren. Darüber hinaus ist die Kollateralversorgung, soweit sie von der A. carotis externa ausgeht, schwer im Vorhinein zu bestimmen, sodass ungewiss bleibt, ob es bei einem evtl. Verschluss der A. ophthalmica zu einer ausreichenden Versorgung des Bulbus über solche Kollateralgefäße kommen kann. Eine Ballon-Test-Okklusion (BTO) der A. carotis interna mit einem Ballon über dem Ursprung der A. ophthalmica ist möglich. Diese Technik kann vor der Coil-Okklusion verwendet werden, um zu prüfen, ob ein akuter Verschluss der A. ophthalmica während der endovaskulären Prozedur evtl. eine unzureichende Blutversorgung des Bulbus zur Folge haben könnte. Ahn et al. [24] verwendeten die BTO bei 14 Patienten, bei denen eine Coil-Okklusion eines paraophthalmischen Aneurysmas mit evtl. Okklusion der A. ophthalmica durchgeführt wurde. Die Autoren beschreiben ihre Untersuchungstechnik, die einen Führungskatheter in der A. carotis communis umfasst. Die Ballonentfaltung erfolgt in Höhe des Ursprungs der A. ophthalmica und dem folgt die Kontrastmittelinjektion über den Führungskatheter, um den 

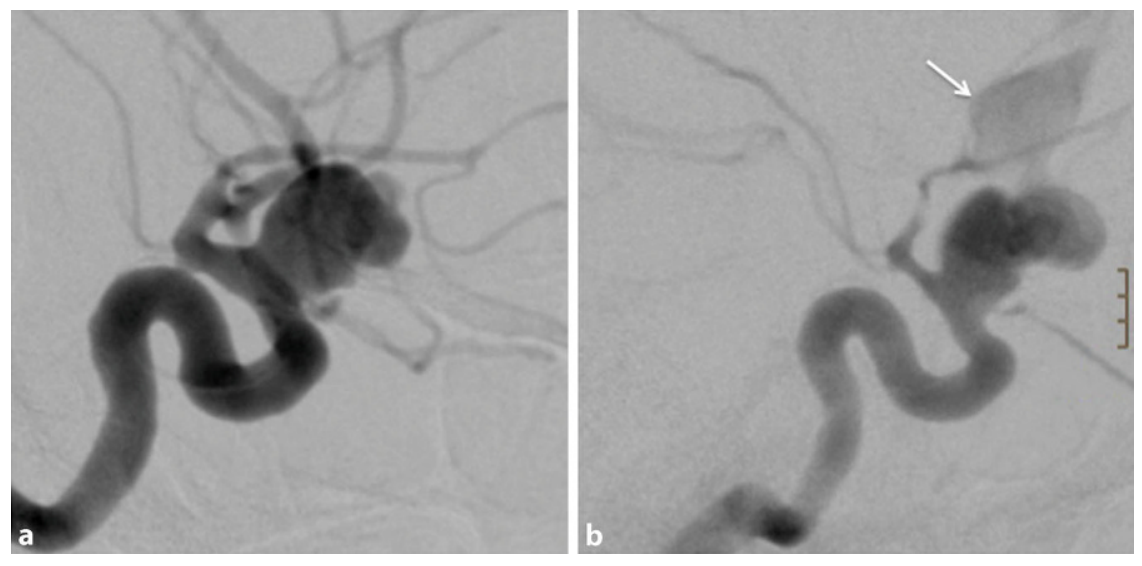

Abb. $3 \Delta$ a Rupturiertes paraophthalmisches Aneurysma bei einer jungen Frau. Eine intraoperative Re-Ruptur trat früh während der mikrochirurgischen Behandlung auf.b Die unmittelbar postoperative digitale Subtraktionsangiographie zeigte eine anhaltende Extravasation (weißer Pfeil) mit einem Anstieg des intrakraniellen Drucks und Unterbrechung der zerebralen Zirkulation. Die Patientin verstarb an den Folgen dieser schweren Blutung trotz einer dekompressiven Kraniektomie
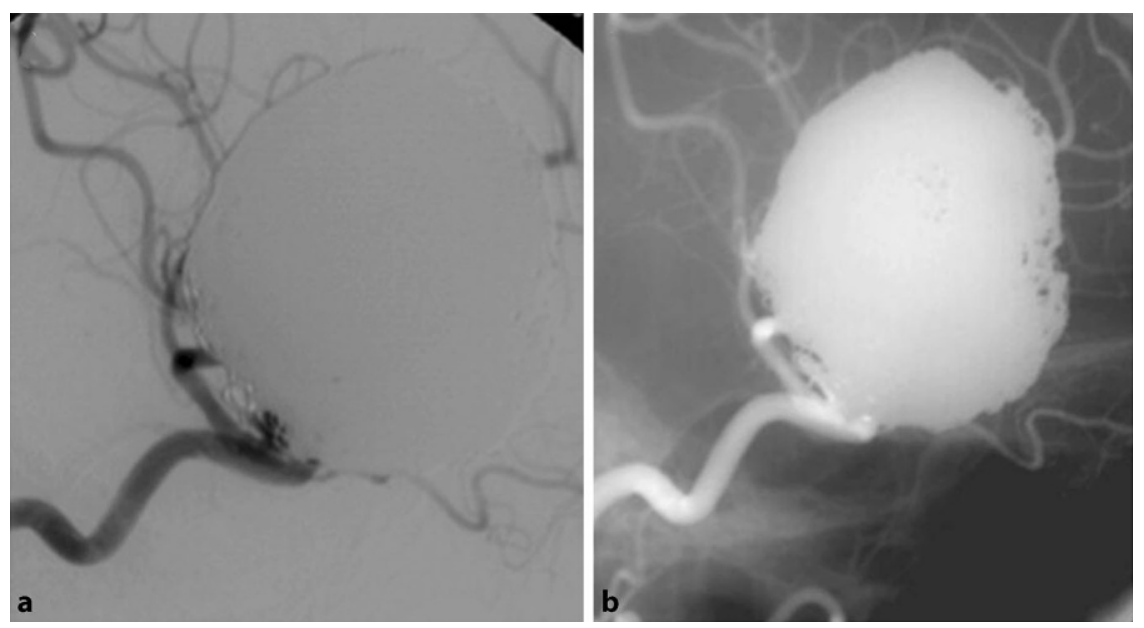

Abb. $4 \Delta$ Riesenhaftes Aneurysma der rechten A. carotis interna mit paraophthalmischem Ursprung $(\mathbf{a}, \mathbf{b})$ bei einer Patientin, die auf dem rechten Auge erblindet war. Der Versuch einer Klippung des Aneurysmas war gescheitert, und die Kollateralversorgung für die rechte A. carotis interna erlaubte keinen Verschluss des Trägergefäßes. Die endovaskuläre Coil-Okklusion des Aneurysmas war vergleichsweise einfach durchführbar. Jedoch waren 9 weitere Behandlungen erforderlich, um eine vollständige und dauerhafte Aneurysmaokklusion zu erzielen

retrograden Zustrom zur A. ophthalmica aus der A. carotis externa sowie den evtl. retinochoroidalen Blush darzustellen. Von den 14 Patienten, bei denen eine BTO durchgeführt wurde, zeigten 12 eine Kollateralversorgung, und 4 dieser Patienten hatten einen Verschluss der A. ophthalmica durch die Coil-Okklusion. Bei keinem dieser Patienten traten nach dem Eingriff Visusstörungen auf.

Allerdings ist das Ergebnis der BTO unter keinen Umständen eine Garantie, dass keine späteren visuellen Komplikationen auftreten. Duxbury et al. [25] berichten den Fall eines Verschlusses der sache geschuldet war, dass die Embolie noch im Krankenhaus aufgetreten war.

In einer kürzlich erschienenen Metaanalyse zeigten Zhu et al. [26] bei 603 ausgewählten Aneurysmen eine vollständige oder nahezu vollständige chirurgische Ausschaltung bei 89,3\%. Stentassistierte Coil-Okklusionen (90,7\%) waren nicht grundsätzlich verschieden, allerdings waren beide Techniken einer reinen Coil-Okklusion mit hier nur $74,6 \%$ deutlich überlegen. Es gab keinen signifikanten Unterschied in der Mortalität der chirurgisch, durch Coil-Okklusion oder durch Stent-assistierte CoilOkklusion behandelten Patienten. Höhere Raten postoperativer intrakranieller Blutungen wurden in der chirurgischen Gruppe beobachtet im Vergleich mit Coil-Okklusion und Stent-assistierter Coil-Okklusion (6,4\% vs. $2,2 \%$ vs. $0 \%$ ). Darüber hinaus waren neurologische Komplikationen häufiger in der operierten Patientengruppe verglichen mit den Patienten, die durch Coil-Okklusion oder Stent-assistierte Coil-Okklusion behandelt wurden ( $23 \%$ vs. $4,9 \%$ vs. $3,9 \%)$.

Der Behandlungsausgang hinsichtlich des Visus war in der operierten Patientengruppe schlechter mit einem ungünstigen Ausgang hinsichtlich des Visus nach Operation bei 5,7\%, bei Stentassistiertem Coiling 1,96\% und bei reiner Coil-Okklusion bei $1 \%$. Diese Daten legen nahe, dass die endovaskuläre Behandlung, bestehend aus Coil-Okklusion mit oder ohne Stentimplantation, Vorteile bietet gegenüber der operativen Behandlung paraophthalmischer Aneurysmen. Die endovaskuläre Versorgung ist heute die Standardbehandlung rupturierter Aneurysmen in der Akutphase nach Subarachnoidalblutung.

Die Einführung von hämodynamisch aktiven Stents (sog. Flow Divertern) in die klinische Praxis brachte einen wesentlichen Fortschritt bei der endovaskulären Behandlung paraophthalmischer Aneurysmen. Diese Implantate führen zu einer Neuausrichtung des Blutstroms weg vom Aneurysma. Nach der Flow Diverter-Implantation kommt es langsam zur Ausbildung einer Intima, die sich über die Stentmaschen ausbreitet und das Trägergefäß im Verlauf der Zeit rekonstru- 

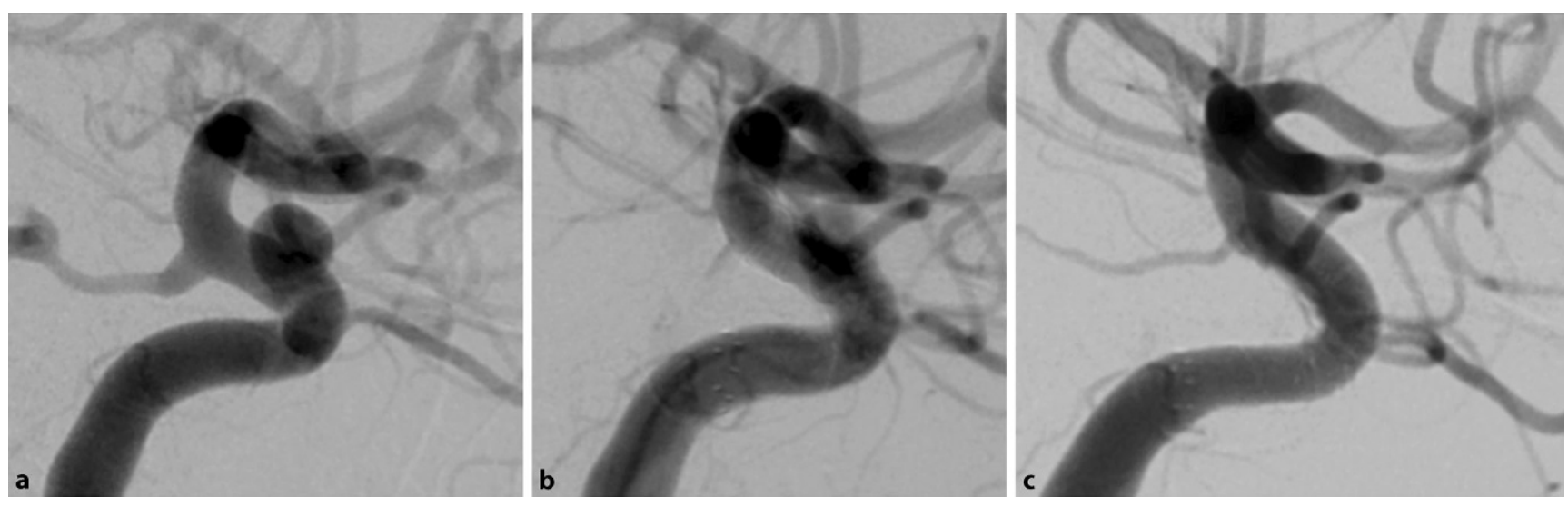

Abb. 5 \ a Endovaskuläre Behandlung eines inzidentellen rechtsseitigen paraophthalmischen Aneurysmas bei einer 41-jährigen Patientin. b Zwei Flow Diverter wurden implantiert. c Die angiographische Nachuntersuchung 3 Monate später bestätigte die vollständige Obliteration des Aneurysmas bei durchgängiger A. ophthalmica
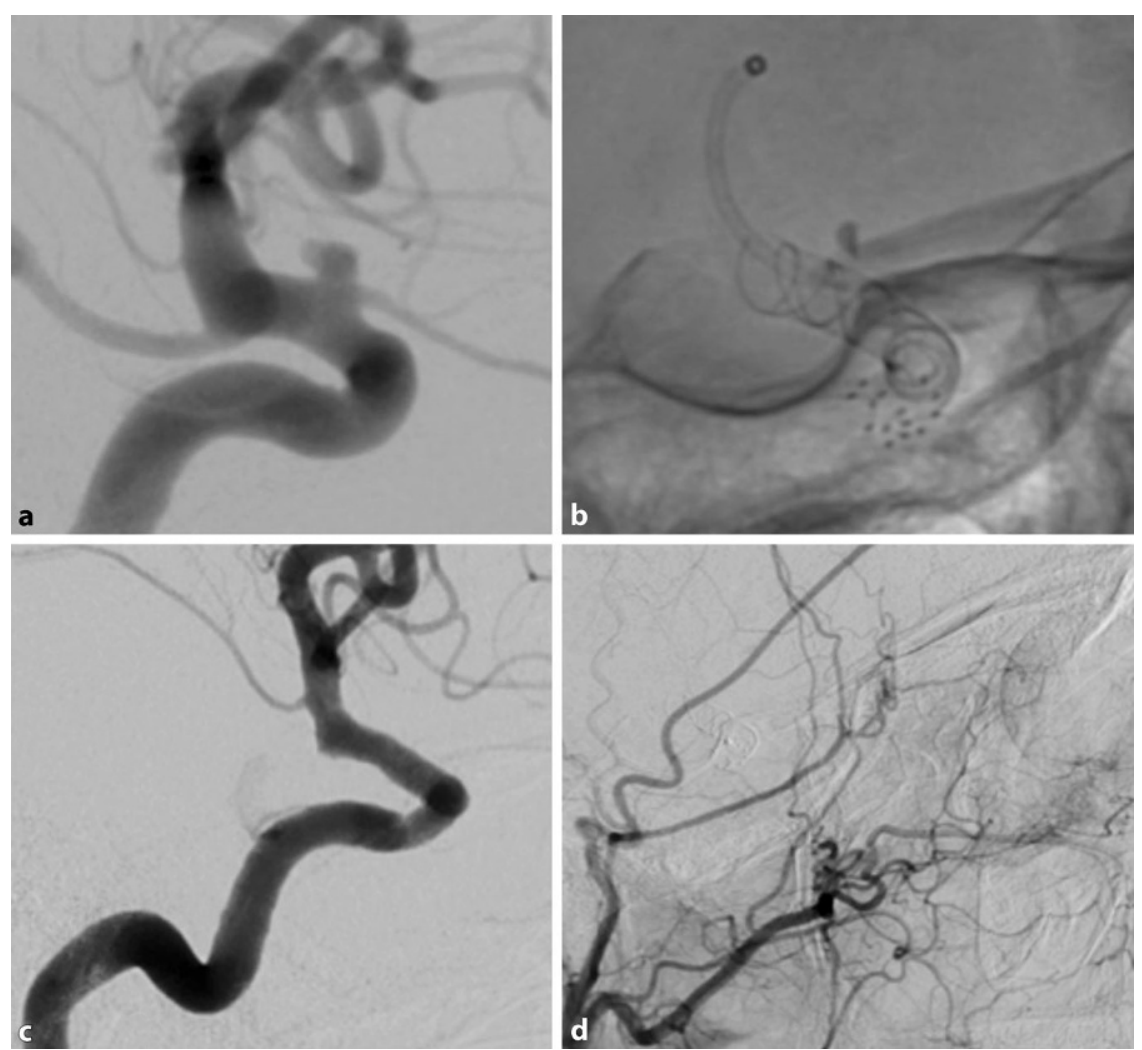

Abb. $6 \Delta$ a Rupturiertes linksseitiges paraophthalmisches Aneurysma, b behandelt mit einem Flow Diverter mit 3,5/15 mm und nachfolgend mit 3/15 mm Größe. c Die angiographische Nachuntersuchung 3 Monate später zeigt die Obliteration von Aneurysma und A. ophthalmica. d Das distale Segment der linken $A$. ophthalmica wurde kollateral von der linken $A$. carotis externa versorgt

iert [27]. Dieser Vorgang kann allerdings mehrere Monate in Anspruch nehmen (॰ Abb. 5).

Mittlerweile haben zahlreiche Studien gezeigt, dass Flow Diverter einen fortschreitenden Aneurysmaverschluss bewirken und mit hoher Sicherheit klinisch eingesetzt werden können [28-46].
Flow Diverter werden bei Patienten in der Akutphase nach Subarachnoidalblutung noch nicht häufig eingesetzt, da sie nur unter dualer Thrombozytenfunktionshemmung sicher implantiert werden können. Dennoch sind sie in der Lage, nicht rupturierte Aneurysmen und Aneurysmarezidive oder Aneurysmareste zu behandeln. Bedauerlicherweise haben nur wenige Registerstudien die Aneurysmalokalisation exakt angegeben. Die oben erwähnte Analyse von Zhu et al. [26] schloss Daten hinsichtlich des Flow Diverter-Einsatzes ein, und die Autoren konnten vergleichbare Verschlussraten für Stent-assistierte Coil-Okklusion und mikrochirurgische Klippung zeigen $(88 \%)$. Die Rate intrazerebraler Blutungen war niedrig und ähnlich zu anderen endovaskulären Techniken, was im Übrigen auch für die neurologischen Komplikationen galt (1,75\%). Es ist interessant, dass kein signifikanter Unterschied hinsichtlich der Rate einer Visusverschlechterung zwischen Flow Diverter-Behandlung, Coil-Okklusion und Stent-assistierter Coil-Okklusion nachgewiesen wurde.

Wie bereits erwähnt, bildet sich NeoIntima auf den Flow Diverter-Maschen, und dies ist ein wichtiger Teilmechanismus beim Verschluss intrakranieller Aneurysmen durch diese Implantate. Bereits früh gab es Bedenken, dass die NeoIntima Seitenäste überdecken und verschließen könnte, was sicher zu einem Verschluss dieser Gefäße führen würde (• Abb. 6).

Puffer et al. [47] berichten über eine Verschlussrate der A. ophthalmica bei $21 \%$ und über $11 \%$ Patienten mit veränderten Flusscharakteristiken der A. ophthalmica nach der Behandlung mit dem Pipeline Embolisation Device (PED, Medtronic, Dublin, Ireland). Trotz dieser hohen Verschlussrate und der häufigen Blutflussveränderung waren in 
dieser Studie alle Patienten asymptomatisch. Ob ein Verschluss von Seitenästen, die mit einem Flow Diverter überdeckt sind, eintritt, hängt davon $a b$, ob es eine distale Kollateralversorgung gibt [48]. Wenn eine distale Kollateralisierung existiert, kommt es zu einem Punkt im Gefäßverlauf, in dem Blutflussgleichgewicht auftreten kann. Das kann dann in einer Flussverlangsamung und letztlich in einem Verschluss des betreffenden, vom Flow Diverter überdeckten Gefäßes proximal dieses Punktes resultieren [49, 50].

In der bislang umfangreichsten Studie zur Flow Diverter-Behandlung paraophthalmischer Aneurysmen fanden Burrows et al. [51] einen Verschluss der A. ophthalmica in ebenfalls $21,6 \%$ aller Patienten, von denen keiner visuelle Symptome entwickelte. Darüber hinaus werteten Rangel-Castilla et al. [52] den Verschluss von Seitenästen aus, die mit einem Flow Diverter überdeckt waren. Von 127 überdeckten Seitenästen (76 davon waren Aa. ophthalmicae) waren 8 (das entspricht $10,5 \%$ ) bei den Nachuntersuchungen mit einer mittleren Nachuntersuchungsdauer von 10 Monaten verschlossen. Die Autoren weisen darauf hin, dass nur die Anzahl der überdeckenden Flow Diverter einen Einfluss auf die Verschlussrate dieser Seitenäste zu haben scheint.

In all diesen Studien wurden keine neuen visuellen Symptome beschrieben.
Es war allerdings nicht eindeutig zu erkennen, wie sorgfältig ophthalmologische Untersuchungen durchgeführt worden waren. Geringe Visusminderungen können ohne Weiteres unbemerkt bleiben. Gesichtsfelddefekte werden bei Konfrontationstesten wie in der Studie von Kerr et al. [53] unter Umständen nicht diagnostiziert, und die Autoren zeigen, dass die Testung des Gesichtsfeldes mit der Konfrontationsmethode nur eine Sensitivität von $39 \%$ insgesamt hat und eine noch geringere Sensitivität von nur $14,3 \%$, wenn es sich um geringe Gesichtsfelddefekte handelt. Insofern ist die standardmäßige klinische Untersuchung, die von einem Nicht-Augenarzt durchgeführt wird, um das Gesichtsfeld zu prüfen, mit einer sicher zu geringen Nachweissensitivität belastet.

Um diese mögliche Unterdiagnostik visueller Komplikationen $\mathrm{zu}$ untersuchen, haben Rouchard et al. [10] eine Studie mit fachophthalmologischer Untersuchung vor und nach der Behandlung von Patienten mit Flow Divertern, die die A. ophthalmica überdecken, durchgeführt. Die Autoren klassifizierten das Aneurysma und die A. ophthalmica hinsichtlich ihrer Lokalisation nach der oben beschriebenen Systematik. Alle Patienten erhielten doppelte Thrombozytenfunktionshemmung 7 Tage vor der Intervention und 3 Monate nach der Behandlung, und ein Augenarzt untersuchte alle Patienten. Bei allen Patienten wurde der Visus bestimmt, und es erfolgten eine Spaltlampenuntersuchung der vorderen Augenabschnitte sowie eine Fluoreszeinuntersuchung, eine Untersuchung in Mydriasis, Tonometrie und die Testung des okulozephalen Reflexes sowie der Lancaster-Test zur Untersuchung der äußeren Augenmuskeln. Wenn eine Auffälligkeit nachgewiesen wurde, erfolgten eine Netzhautuntersuchung und eine Fluoreszeinangiographie. Bei $80 \%$ der Patienten mit einer Typ-A-Konfiguration (die A. ophthalmica entspringt aus dem Aneurysmafundus) traten visuelle Symptome nach der Behandlung auf. Diese waren Folge retinaler Embolien und in $85 \%$ dauerhaft. Bei allen Patienten mit einer Typ-A-Morphologie blieb die A. ophthalmica durchgängig, und es ist davon auszugehen, dass die retinalen Embolien durch eine Thrombusbildung im Aneurysmafundus entstanden sind mit einer möglichen Fragmentierung des Thrombus, der dann dem Gefäßverlauf der durchbluteten A. ophthalmica folgte. Die Autoren beschrieben auch eine hohe Rate ischämischer Atrophien des N. opticus bei Typ-C-Aneurysmen, bei denen die A. ophthalmica auf der konvexen Seite der A. carotis interna entspringt. Es wurde vermutet, dass dies mit der geringeren Zellengröße des Stents zusammenhängt, die an der inneren Kurve des geflochtenen Implantats entsteht, zusammen mit der Kompression des Implantats entlang der konvexen Fläche

Hier steht eine Anzeige. 

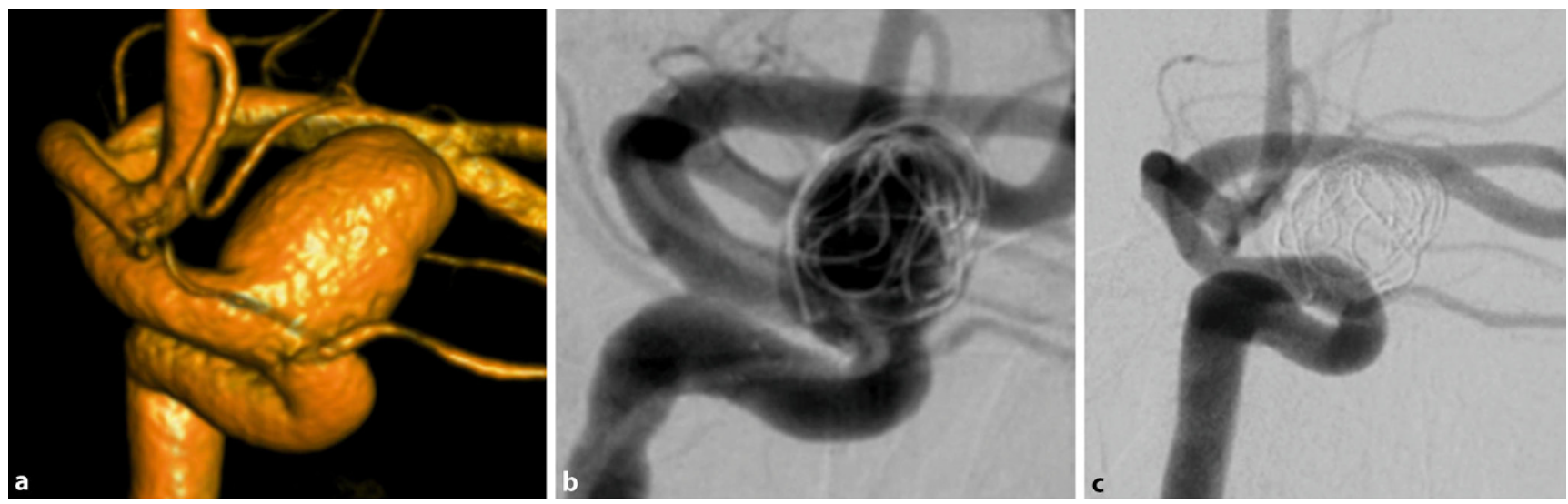

Abb. $7 \Delta$ a Großes paraophthalmisches Aneurysma bei einer 41-jährigen Patientin mit zunehmender Sehstörung des linken Auges. b Eine Kombination von 2 intrasakkulären Flow-Divertern (Medina, 9/15 Framing, 8/10 Framing, Medtronic, Dublin, Ireland) und 2 extrasakkulären Flow-Divertern (p64, 4/18, 5/21, phenox $\mathrm{GmbH}$, Bochum, Deutschland) wurden in einer Behandlungssitzung implantiert. c Dies führte zu einer vollständigen Ausschaltung des Aneurysmas und zu einer deutlichen Verbesserung des Visus

des Implantat-tragenden Gefäßes. Dies könnte zu vermindertem Blutfluss und dadurch zu einer Ischämie des N. opticus führen. Diese Aussagen der Studie bieten wichtige Informationen zu den möglichen Komplikationen der Flow DiverterBehandlung paraophthalmischer Aneurysmen und betrachtet Details der unterschiedlichen Typen von Komplikationen in ihrem Bezug zu unterschiedlichen anatomischen Ausgangssituationen.

\section{I) Große Aneurysmen können durch ihre Raumforderung Hirnnervenparesen verursachen}

Viele Patienten werden ohne Symptome, die auf ihre Aneurysmen zu beziehen wären, vorstellig, es handelt sich insofern um inzidentell nachgewiesene Aneurysmen. Große Aneurysmen können dagegen durch ihre Raumforderung Hirnnervenparesen verursachen [54]. Hier entsteht ein Behandlungsdilemma insofern, als die Coil-Okklusion zwar ein weiteres Größenwachstum verhindern kann und einer möglichen Ruptur des Aneurysmas vorbeugt, jedoch den raumfordernden Effekt nicht beseitigt und unter Umständen sogar noch vergrößert. Diese Aneurysmen sind häufig groß und haben einen breiten Aneurysmahals, und dadurch ist die Coil-Okklusion unvollständig, und es kommt zu einer hohen Rate von Aneurysmarezidiven [54]. Unter diesen Umständen haben sich Flow
Diverter besonders bewährt. Szikora et al. [55] veröffentlichten 30 Patienten mit Aneurysmen mit einem Durchmesser von mehr als $10 \mathrm{~mm}$, die raumforderungsbedingte Symptome aufwiesen. Sie konnten zeigen, dass magnetresonanztomographisch ein Schrumpfen bei allen Aneurysmen nachgewiesen wurde und dass sich $90 \%$ vollständig zurückgebildet hatten. In ähnlicher Weise kam es bei 6 Patienten, die initial eine Visusminderung aufwiesen, zu einer Visusverbesserung im Beobachtungszeitraum. Wiederum ähnliche Ergebnisse wurden bei 10 Patienten gesehen, die sich mit Doppelbildern vorstellten, von denen 7 eine vollständige Rückbildung, 3 nur geringe Residuen ihrer Doppelbilder im Verlauf erfuhren. Diese günstigen Effekte der Flow-Diverter-Behandlung basieren nicht nur auf der Reduktion der Größe des Aneurysmas, sondern auch auf der verminderten Aneurysmapulsation, die auf die umgebenden Strukturen weitergeleitet wird. So konnten kürzlich Binyamin et al. [56] die Rückbildung einer Okulomotoriusparese nach Flow Diverter-Behandlung nachweisen, obwohl die Aneurysmagröße unverändert blieb. Patel et al. [57] berichten in einem ähnlichen Fall über eine Visusverbesserung nach bilateralem Visusverlust, erzielt durch die FlowDiverter-Behandlung eines riesenhaften paraophthalmischen Aneurysmas. Diese Autoren gehen davon aus, dass sowohl die verminderte Raumforderung als auch die verminderte Aneurysmapulsation zu diesem Effekt beigetragen haben.

Der Behandlungszeitraum spielt eine wichtige Rolle. Brown et al. [58] zeigten, dass alle Patienten, die mit einer Hirnnervenparese innerhalb eines Monats behandelt worden waren, eine vollständige Rückbildung ihrer Symptome erlebten, während dies nur bei $44,4 \%$ der Patienten der Fall war, die nach 1 Monat und länger seit Symptombeginn behandelt worden waren.

Unter gewissen Umständen sollten große Aneurysmen mit einem Durchmesser von mehr als $10 \mathrm{~mm}$ auch intrasakkulär und nicht nur mit einem extrasakkulären Flow Diverter behandelt werden (•Abb. 7). Aneurysmen mit einem Durchmesser von 10 und mehr Millimetern können nach der alleinigen Flow Diverter-Behandlung [59] rupturieren, und aus diesem Grunde ist die vorhergehende Coil-Okklusion zu erwägen. Neben den herkömmlichen ablösbaren Platinmikrospiralen können auch das Medina Embolic Device (MED, Medtronic) oder die Woven EndoBridge (WEB, Microvention, Aliso Viejo, CA, USA) mit intraluminalen/ extrasakkulären Flow Divertern kombiniert werden [60]. Darüber hinaus muss beachtet werden, dass die Ausbildung eines intraaneurysmatischen Thrombus, wie sie durch die Flow Diverter-Implantation verursacht werden kann, zu einer Zunahme der Raumforderungswirkung führen kann und dadurch, zumindest 
vorübergehend, die okulären Symptome zunehmen können. Dies wird bei bis zu $15 \%$ der Patienten beobachtet [58], und diese Patienten sollten zumindest kurzfristig Kortikosteroide erhalten.

\section{Fazit für die Praxis}

\section{- In neurointerventionellen Fachab- teilungen sind paraophthalmische Aneurysmen keineswegs selten. \\ - Das Management dieser Aneurysmen ist mit spezifischen Risiken behaftet. Eine sorgfältige Abwägung vor jeder Intervention ist erforderlich. \\ - Auch wenn Coil-Okklusion und Stent- assistierte Coil-Okklusion die Grund- lage für die Behandlung rupturierter Aneurysmen darstellen, kommen Flow Diverter-Implantationen für nicht rupturierte Aneurysmen heute in Betracht. \\ - Die Komplikationsraten dieser Pro- zeduren sind insgesamt niedrig, mögliche visuelle Komplikationen müssen jedoch im Voraus bedacht, und die Patienten müssen auf diese Gefahr hingewiesen werden.}

\section{Korrespondenzadresse}

\section{P. Bhogal}

Neuroradiologische Klinik, Neurozentrum, Klinikum Stuttgart

Kriegsbergstr. 60, 70174 Stuttgart, Deutschland bhogalweb@aol.com

\section{Einhaltung ethischer Richtlinien}

Interessenkonflikt. P. Bhogal und M. Aguilar Pérez sind Proktoren und Berater der Firma phenox $\mathrm{GmbH}$ mit angemessener finanzieller Vergütung. H. Henkes ist Mitgründer und Miteigentümer der phenox $\mathrm{GmbH}$. G. Sauder, H. Bäzner und O. Ganslandt geben an, dass kein Interessenkonflikt besteht.

Es handelt sich um eine Literaturbetrachtung, für die eine berufsrechtliche Beratung nicht erforderlich ist.

Open Access. Dieser Artikel wird unter der Creative Commons Namensnennung 4.0 International Lizenz (http://creativecommons.org/licenses/by/4.0/deed. de) veröffentlicht, welche die Nutzung, Vervielfältigung, Bearbeitung, Verbreitung und Wiedergabe in jeglichem Medium und Format erlaubt, sofern Sie den/die ursprünglichen Autor(en) und die Quelle ordnungsgemäß nennen, einen Linkzur Creative Commons Lizenz beifügen und angeben, ob Änderungen vorgenommen wurden.

\section{Literatur}

1. Rinkel GJ, Djibuti M, Algra A, van Gijn J (1998) Prevalence and risk of rupture of intracranial aneurysms: a systematic review. Stroke 29:251-256

2. Molyneux A, Kerr R, Stratton I et al (2002) International Subarachnoid Aneurysm Trial (ISAT) of neurosurgical clipping versus endovascular coiling in 2143 patients with ruptured intracranial aneurysms: a randomised trial. Lancet 360:1267-1274

3. Coon AL, Paul AR, Colby GP et al (2011) Comparison of tertiary-center aneurysm location frequencies in 400 consecutive cases: decreasing incidence of posterior communicating artery region aneurysms. Surg Neurol Int. doi:10.4103/2152-7806. 86832

4. Forget TR, Benitez R, Veznedaroglu E et al (2001) A review of size and location of ruptured intracranial aneurysms. Neurosurgery 49:1322-1325 (discussion 1325-1326)

5. Jeong Y-G, Jung Y-T, Kim M-S et al (2009) Size and location of ruptured intracranial aneurysms. J Korean Neurosurg Soc 45:11-15. doi:10.3340/ jkns.2009.45.1.11

6. Wiebers DO, Whisnant JP, Huston J et al (2003) Unruptured intracranial aneurysms: natural history, clinical outcome, and risks of surgical and endovascular treatment. Lancet 362:103-110

7. UCAS Japan Investigators, Morita A, Kirino T et al (2012) The natural course of unruptured cerebral aneurysms in a Japanese cohort. N Engl J Med 366:2474-2482. doi:10.1056/NEJMoa1113260

8. Ferguson GG, Drake CG (1980) Carotid-ophthalmic aneurysms: the surgical management of those cases presenting with compression of the optic nerves and chiasm alone. Clin Neurosurg 27:263-307

9. Bouthillier A, van Loveren HR, Keller JT (1996) Segments of the internal carotid artery: a new classification. Neurosurgery 38:425-432 (discussion 432-433.)

10. Rouchaud A, Leclerc O, Benayoun $Y$ et al (2015) Visual outcomes with flow-diverter stents covering the ophthalmic artery for treatment of internal carotid artery aneurysms. AJNR Am J Neuroradiol 36:330-336. doi:10.3174/ajnr.A4129

11. Boet R, Wong GKC, Poon WS et al (2005) Aneurysm recurrence after treatment of paraclinoid/ ophthalmic segment aneurysms - a treatmentmodality assessment. Acta Neurochir (Wien) 147:611-616. doi:10.1007/s00701-005-0524-4 (discussion 616)

12. Sengupta RP, Gryspeerdt GL, Hankinson J (1976) Carotid-ophthalmic aneurysms. J Neurol Neurosurg Psychiatry 39:837-853

13. Kumon Y, Sakaki S, Kohno K et al (1997) Asymptomatic, unruptured carotid-ophthalmic artery aneurysms: angiographical differentiation of each type, operative results, and indications. Surg Neurol 48:465-472

14. Raco A, Frati A, Santoro A et al (2008) Longterm surgical results with aneurysms involving the ophthalmic segment of the carotid artery. J Neurosurg 108:1200-1210. doi:10.3171/JNS/ 2008/108/6/1200

15. Ng PY, Huddle D, Gunel M, Awad IA (2000) Intraoperative endovascular treatment as an adjunct to microsurgical clipping of paraclinoid aneurysms.J Neurosurg 93:554-560. doi:10.3171/ jns.2000.93.4.0554

16. Day AL (1990) Aneurysms of the ophthalmic segment. A clinical and anatomical analysis.
J Neurosurg 72:677-691. doi:10.3171/jns.1990.72. 5.0677

17. Barami K, Hernandez VS, Diaz FG, Guthikonda M (2003) Paraclinoid carotid aneurysms: surgical management, complications, and outcome based on a new classification scheme. Skull Base 13:31-41. doi:10.1055/s-2003-820555

18. Ferguson GG, Drake CG (1981) Carotid-ophthalmic aneurysms: visual abnormalities in 32 patients and the results of treatment. Surg Neurol 16:1-8

19. Roy D, Raymond J, Bouthillier A et al (1997) Endovascular treatment of ophthalmic segment aneurysms with Guglielmi detachable coils. AJNR Am J Neuroradiol 18:1207-1215

20. Yavuz K, Geyik S, Pamuk AG et al (2007) Immediate and midterm follow-up results of using an electrodetachable, fully retrievable SOLO stent system in the endovascular coil occlusion of wide-necked cerebral aneurysms. J Neurosurg 107:49-55. doi:10.3171/JNS-07/07/0049

21. Sun Y, Li Y, Li A (2011) Endovascular treatment of paraclinoid aneurysms. Interv Neuroradiol 17:425-430

22. Kim B, Jeon P, Kim K et al (2016) Endovascular treatment of unruptured ophthalmic artery aneurysms: clinical usefulness of the balloon occlusion test in predicting vision outcomes after coil embolization. J Neurointerv Surg 8:696-701. doi:10.1136/neurintsurg-2015-011800

23. Lin N, Brouillard AM, Xiang J et al (2015) Endovascular management of adjacent tandem intracranial aneurysms: utilization of stentassisted coiling and flow diversion. Acta Neurochir (Wien) 157:379-387. doi:10.1007/s00701-0142318-z

24. Ahn JH, Cho YD, Kang H-Setal (2014) Endovascular treatment of ophthalmic artery aneurysms: assessing balloon test occlusion and preservation of vision in coil embolization. AJNR Am J Neuroradiol 35:2146-2152. doi:10.3174/ajnr. A3999

25. Duxbury O, Bhogal P, Cloud G, Madigan J (2014) Successful treatment of central retinal artery thromboembolism with ocular massage and intravenous acetazolamide. BMJ Case Rep. doi:10. 1136/bcr-2014-207943

26. Zhu Y, Pan J, Shen J et al (2015) Clinical and radiological outcomes after treatment of unruptured paraophthalmic internal carotid artery aneurysms: a comparative and pooled analysis of single-center experiences. World Neurosurg 84:1726-1738. doi:10.1016/j.wneu.2015.07.036

27. Kadirvel R, Ding Y-H, Dai D et al (2014) Cellular mechanisms of aneurysm occlusion after treatment with a flow diverter. Radiology 270:394-399. doi:10.1148/radiol.13130796

28. Berge J, Biondi A, Machi P et al (2012) Flowdiverter silk stent for the treatment of intracranial aneurysms: 1-year follow-up in a multicenter study. AJNR Am J Neuroradiol 33:1150-1155. doi:10.3174/ajnr.A2907

29. Wagner A, Cortsen M, Hauerberg J et al (2012) Treatment of intracranial aneurysms. Reconstruction of the parent artery with flow-diverting (Silk) stent. Neuroradiology 54:709-718. doi:10.1007/ s00234-011-0949-9

30. Maimon S, Gonen L, Nossek E et al (2012) Treatment of intra-cranial aneurysms with the SILK flow diverter: 2 years' experience with 28 patients at a single center. Acta Neurochir (Wien) 154:979-987.doi:10.1007/s00701-012-1316-2

31. Lubicz B, Van der Elst O, Collignon Let al (2015) Silk flow-diverter stent for the treatment of intracranial aneurysms: a series of 58 patients with emphasis 
on long-term results. AJNR Am J Neuroradiol 36:542-546. doi:10.3174/ajnr.A4143

32. Strauss I, Maimon S (2016) Silk flow diverter in the treatment of complex intracranial aneurysms: a single-center experience with 60 patients. Acta Neurochir (Wien) 158:247-254. doi:10.1007/ s00701-015-2644-9

33. Mpotsaris A, Skalej M, Beuing O et al (2015) Longterm occlusion results with SILK flow diversion in 28 aneurysms: do recanalizations occur during follow-up? Interv Neuroradiol 21:300-310. doi:10. 1177/1591019915583119

34. Shankar JJS, Tampieri D, lancu D et al (2016) SILK flow diverter for complex intracranial aneurysms: a Canadian registry. J Neurointerv Surg 8:273-278. doi:10.1136/neurintsurg-2015-011708

35. Briganti F, Napoli M, Tortora F et al (2012) Italian multicenter experience with flow-diverter devices for intracranial unruptured aneurysm treatment with periprocedural complications a retrospective data analysis. Neuroradiology 54:1145-1152. doi:10.1007/s00234-012-1047-3

36. Yu SC-H, Kwok C-K, Cheng P-W et al (2012) Intracranial aneurysms: midterm outcome of pipeline embolization device - a prospective study in 143 patients with 178 aneurysms. Radiology 265:893-901.doi:10.1148/radiol.12120422

37. O'Kelly CJ, Spears J, Chow M et al (2013) Canadian experience with the pipeline embolization device for repair of unruptured intracranial aneurysms. AJNR Am J Neuroradiol 34:381-387. doi:10.3174/ ajnr.A3224

38. Saatci I, Yavuz K, Ozer C et al (2012) Treatment of intracranial aneurysms using the pipeline flowdiverter embolization device: a single-center experience with long-term follow-up results. AJNR Am J Neuroradiol 33:1436-1446. doi:10.3174/ajnr. A3246

39. Fischer S, Vajda Z, Aguilar Perez M et al (2012) Pipeline embolization device (PED) for neurovascular reconstruction: initial experience in the treatment of 101 intracranial aneurysms and dissections. Neuroradiology 54:369-382. doi:10.1007/s00234011-0948-x

40. Wakhloo AK, Lylyk P, de Vries J et al (2015) Surpass flow diverter in the treatment of intracranial aneurysms: a prospective multicenter study. Am J Neuroradiol 36:98-107. doi:10.3174/ajnr.A4078

41. De Vries J, Boogaarts J, Van Norden A, Wakhloo AK (2013) New generation of flow diverter (surpass) for unruptured Intracranial aneurysms: a prospective single-center study in 37 patients. Stroke 44:1567-1577. doi:10.1161/STROKEAHA. 111.000434

42. Möhlenbruch MA, Herweh $C$, Jestaedt $L$ et al (2015) The FRED flow-diverter stent for intracranial aneurysms: clinical study to assess safety and efficacy. AJNR Am J Neuroradiol 36:1155-1161. doi:10.3174/ajnr.A4251

43. Poncyljusz W, Sagan L, Safranow K, Rać M (2013) Initial experience with implantation of novel dual layer flow-diverter device FRED. Wideochir Inne Tech Maloinwazyjne 8:258-264. doi:10.5114/ wiitm.2011.35794

44. Briganti F, Leone G, Ugga L et al (2016) Midterm and long-term follow-up of intracranial aneurysms treated by the $\mathrm{p} 64$ flow modulation device: a multicenter experience. J Neurointerv Surg. doi:10.1136/neurintsurg-2016-012502

45. Briganti F, Leone G, Ugga L et al (2016) Safety and efficacy of flow re-direction endoluminal device (FRED) in the treatment of cerebral aneurysms: a single center experience. Acta Neurochir (Wien). doi:10.1007/s00701-016-2875-4
46. Kocer N, Islak C, Kizilkilic O et al (2014) Flow Redirection Endoluminal Device in treatment of cerebral aneurysms: initial experience with short-term follow-up results. J Neurosurg 120:1158-1171. doi:10.3171/2014.1.JNS131442

47. Puffer RC, Kallmes DF, Cloft HJ, Lanzino G (2012) Patency of the ophthalmic artery after flow diversion treatment of paraclinoid aneurysms. J Neurosurg 116:892-896. doi:10.3171/2011.11. JNS111612

48. Kulcsár Z, Ernemann U, Wetzel SG et al (2010) High-profile flow diverter (silk) implantation in the basilar artery: efficacy in the treatment of aneurysms and the role of the perforators. Stroke 41:1690-1696. doi:10.1161/STROKEAHA. 110.580308

49. Albuquerque FC (2012) Visual impairment after endovascular treatment: does stent placement increase the incidence? World Neurosurg 78:222. doi:10.1016/j.wneu.2012.01.015

50. Fiorella D, Lylyk P, Szikora I et al (2009) Curative cerebrovascular reconstruction with the Pipeline embolization device: the emergence of definitive endovascular therapy for intracranial aneurysms. J Neurointerv Surg 1:56-65. doi:10.1136/jnis.2009. 000083

51. Burrows AM, Brinjikji W, Puffer RC et al (2016) Flow diversion for ophthalmic artery aneurysms. AJNR Am J Neuroradiol. doi:10.3174/ajnr.A4835

52. Rangel-Castilla L, Munich SA, Jaleel N et al (2016) Patency of anterior circulation branch vessels after pipeline embolization: longer-term results from 82 aneurysm cases. J Neurosurg. doi:10.3171/2016.4. JNS16147

53. Kerr NM, Chew SSL, Eady EK et al (2010) Diagnostic accuracy of confrontation visual field tests. Neurology 74:1184-1190. doi:10.1212/WNL. Ob013e3181d90017

54. van Rooij WJ, Sluzewski M (2008) Unruptured large and giant carotid artery aneurysms presenting with cranial nerve palsy: comparison of clinical recovery after selective aneurysm coiling and therapeutic carotid artery occlusion. AJNR Am J Neuroradiol 29:997-1002. doi:10.3174/ajnr.A1023

55. Szikora I, Marosfoi M, Salomváry B et al (2013) Resolution of mass effect and compression symptoms following endoluminal flow diversion for the treatment of intracranial aneurysms. AJNR Am J Neuroradiol 34:935-939. doi:10.3174/ajnr. A3547

56. Binyamin TR, Dahlin BC, Waldau B (2016) Resolution of third nerve palsy despite persistent aneurysmal mass effect after flow diversion embolization of posterior communicating artery aneurysms. J Clin Neurosci 31:207-209. doi:10. 1016/j.jocn.2016.02.027

57. Patel S, Fargen KM, Peters K et al (2014) Return of visual function after bilateral visual loss following flow diversion embolization of a giant ophthalmic aneurysm due to both reduction in mass effect and reduction in aneurysm pulsation. BMJ Case Rep. doi:10.1136/bcr-2013-010960

58. Brown BL, Lopes D, Miller DA et al (2016) The fate of cranial neuropathy after flow diversion for carotid aneurysms. J Neurosurg 124:1107-1113. doi:10. 3171/2015.4.JNS142790

59. Kulcsár Z, Houdart E, Bonafé A et al (2011) Intraaneurysmal thrombosis as a possible cause of delayed aneurysm rupture after flow-diversion treatment. AJNR Am J Neuroradiol 32:20-25. doi:10.3174/ajnr.A2370

60. Aguilar PM, Bhogal P, Martinez Moreno R et al (2016) The Medina Embolic Device: early clinical experience from a single center. J Neurointerv Surg. doi:10.1136/neurintsurg-2016-012539
Ärzte wollen digitalen Austausch

Das Gros der Ärzte wünscht sich digitale Kommunikation zwischen Kliniken und Praxen. Dies zeigt die zur Jahreswende veröffentlichte Studie „Ärzte im Zukunftsmarkt Gesundheit 2017“. Die Stiftung Gesundheit hatte sowohl niedergelassene Ärzte als auch leitende Klinikärzte zur transsektoralen Zusammenarbeit befragt. 36,4 Prozent der niedergelassenen Ärzte würden laut der Studie am liebsten per E-Mail in sicherer Umgebung kommunizieren, 21,5 Prozent über ein IT-System, das sich idealerweise ins Arztinformationssystem integrieren ließe. Auch bei den Klinikärzten stehen diese beiden Kommunikationswege ganz oben auf der Wunschliste: 40,4 Prozent wünschen sich ein IT-System, 38,5 Prozent würden gern E-Mails in sicherer Umgebung nutzen.

Tatsächlich kommunizieren Praxen und Kliniken allerdings immer noch vorwiegend per Brief: Fast 60 Prozent der niedergelassenen Ärzte und sogar über 80 Prozent der Klinikärzte nutzen heute diesen Weg. Wenn sie die Wahl hätten, würden sich aber nur 18,7 Prozent der Ärzte und nur 11,5 Prozent der Klinikärzte für diesen herkömmlichen Weg entscheiden.

Nachbarländer in Europa zeigen dagegen bereits in der Praxis, wie verbesserte Kommunikations- und Informationsstrukturen aussehen können.

Quelle: Stiftung Gesundheit 